\title{
Effect of ozone oxidative preconditioning on oxidative stress injury in a rat model of kidney transplantation
}

\author{
TAO QIU ${ }^{1 *}$, ZHI-SHUN WANG ${ }^{2}$, XIU-HENG LIU ${ }^{2}$, HUI CHEN $^{2}$, \\ JIANG-QIAO ZHOU ${ }^{1}$, ZHI-YUAN CHEN ${ }^{2}$, MIN WANG ${ }^{2}$, GUAN-JUN JIANG ${ }^{2}$, LEI WANG ${ }^{2}$, GANG YU $^{2}$, \\ LONG ZHANG $^{2}$, YE SHEN ${ }^{2}$, LU ZHANG ${ }^{2}$, LI HE $^{2}$, HUA-XIN WANG $^{3}$ and WEN-JING ZHANG ${ }^{4}$ \\ Departments of ${ }^{1}$ Organ Transplantation, ${ }^{2}$ Urology and ${ }^{3}$ Anesthesiology, \\ Renmin Hospital of Wuhan University, Wuhan, Hubei 430060; ${ }^{4}$ Department of Infectious Diseases, Union Hospital, \\ Tongji Medical College, Huazhong University of Science and Technology, Wuhan, Hubei 430022, P.R. China
}

Received December 20, 2015; Accepted December 23, 2016

DOI: $10.3892 / \mathrm{etm} .2017 .4193$

\begin{abstract}
The aim of the present study was to investigate the protective effect of ozone oxidative preconditioning (OOP) on renal oxidative stress injury in a rat model of kidney transplantation. Thirty-six male Sprague Dawley (SD) rats were randomly divided into three groups: A sham (S) group, a kidney transplantation (KT) group and an OOP and kidney transplantation $(\mathrm{OOP}+\mathrm{KT})$ group. In the $\mathrm{S}$ group, the rats' abdomens were opened and closed without transplantation. In the KT group, the rats received a left kidney from donor SD rats. In the OOP+KT group, donor SD rats received 15 OOP treatments by transrectal insufflations $(1 \mathrm{mg} / \mathrm{kg})$, once a day, at an ozone concentration of $50 \mu \mathrm{g} / \mathrm{ml}$, before the kidney transplantation. Twenty-four hours after transplantation, the parameters of renal function of the recipients were measured. The morphology and pathological effects of renal allograft were examined using hematoxylin and eosin staining, periodic acid-Schiff staining, a terminal deoxynucleotidyl transferase dUTP nick end labeling assay and immunohistochemistry. Markers of oxidative stress were also detected using the thiobarbituric acid method, and expression levels of Nrf-2 and HO-1 were determined by western blot analysis. Blood urea nitrogen and creatinine levels were significantly decreased in the OOP $+\mathrm{KT}$ group compared with the KT group, and the morphology and pathological changes of renal allograft were also less severe. Meanwhile, the renal allograft cell apoptosis index was significantly higher in the KT group compared to the OOP+KT group
\end{abstract}

Correspondence to: Professor Xiu-Heng Liu, Department of Urology, Renmin Hospital of Wuhan University, 238 Jiefang Road, Wuhan, Hubei 430060, P.R. China

E-mail: xhliu2014@126.com

*Contributed equally

Key words: kidney transplantation, ozone, nuclear factor erythroid 2-related factor 2 , heme oxygenase 1 , oxidative stress
$(\mathrm{P}<0.05)$. Levels of superoxide dismutase, glutathione and catalase in the renal allografts were significantly higher in the OOP+KT group compared to those in the KT group $(\mathrm{P}<0.05)$, while malondialdehyde levels were significantly lower in the OOP+KT group compared to those in the KT group $(\mathrm{P}<0.05)$. Western blot analysis indicated that the expression levels of nuclear factor erythroid 2-related factor 2 (Nrf-2) and heme oxygenase $1(\mathrm{HO}-1)$ were significantly higher in the $\mathrm{OOP}+\mathrm{KT}$ compared to the KT group $(\mathrm{P}<0.05)$. In conclusion, the mechanism by which OOP alleviates oxidative stress injury in renal transplantation may be related to the activation of the signaling pathways of Nrf-2/HO-1 and inhibition of renal tubular epithelial cell apoptosis.

\section{Introduction}

Kidney transplantation is the most effective treatment for end-stage renal disease in patients who require dialysis, helping to improve their quality of life and prolong their survival (1). There are several immunosuppressive agents currently used for renal transplantation, which are effective at preventing acute rejection throughout the transplantation process. However, the process still has some adverse effects, including ischemia/reperfusion injury (IRI), which occurs after blood flow recovery and restoration to tissues and organs. It has previously been identified that IRI is associated with an increased incidence of acute rejection and decreased long-term allograft survival, and is considered to be one of the leading causes of early allograft dysfunction $(2,3)$. Therefore, it is necessary to adopt effective measures to reduce or alleviate IRI during kidney transplantation.

Ozone is a powerful oxidizing gas, which is commonly used as a disinfectant in the water and food industries (4). Although ozone is a potentially toxic agent, it can modulate many biochemical pathways at low and controlled non-toxic doses (5). Medical ozone therapy involves delivering a mixture of gaseous ozone and oxygen to the body (6). It has previously been identified that ozone therapy can be useful in treating inflammation-mediated diseases, such as infected wounds, burns, and advanced ischemic diseases (7). Ozone therapy also results in resistance to oxidative stress by inducing 
antioxidant systems $(8,9)$. Furthermore, it has been reported that ozone oxidative preconditioning (OOP), a type of ozone therapy (10) has a protective effect against IRI in the liver and kidney $(11,12)$. To our knowledge, the current study is the first to investigate the effect of OOP on IRI in a homologous kidney transplantation in a rat model.

\section{Materials and methods}

Animal preparation. The experimental protocol used in the present study was approved by the Animal Ethics Review Committee of Wuhan University (Wuhan, China), and the procedures were conducted in accordance with the recommendations in the Guide for the Care and Use of Laboratory Animals from the National Institutes of Health. A total of 36 clean male, 7-week-old, Sprague Dawley (SD) rats (250-300 g) were purchased from Huazhong University of Science and Technology, all of them were kept in an air-filtered, homeothermal $\left(20-22^{\circ} \mathrm{C}\right)$, and light-controlled (light between 7:00 a.m. and 7:00 p.m.) room, and allowed free access to a standard diet.

Experimental protocol. Kidney donor rats were randomly divided into three groups, 12 rats in each, with 6 donors and 6 recipients. In the OOP and kidney transplantation $(\mathrm{OOP}+\mathrm{KT})$ group, donor rats $(\mathrm{n}=6)$ received 15 OOP treatments by transrectal insufflations $(1 \mathrm{mg} / \mathrm{kg})$, once a day, at an ozone concentration of $50 \mu \mathrm{g} / \mathrm{ml}$, before their left kidneys were transplanted into recipient rats. In the kidney transplantation (KT) group, recipient rats each received a left kidney from a donor rat that had not undergone OOP treatment. The experiments that follow were only on the donated kidneys in groups $\mathrm{OOP}+\mathrm{KT}(\mathrm{n}=6)$ and KT $(\mathrm{n}=6)$. In the sham (S) group $(\mathrm{n}=12)$, the rats' abdomens were opened and closed without transplantation; six kidneys from six rats were used in the subsequent experiments.

Surgical procedure. In all groups, donors were injected intraperitoneally with atropine $(0.01 \mathrm{mg} / \mathrm{kg})$, buprenorphine $(0.04 \mathrm{mg} / \mathrm{kg})$ and diazepam $(10 \mathrm{mg} / \mathrm{kg})$, all drugs were purchased from Sigma-Aldrich; Merck Millipore (Darmstadt, Germany). After $10 \mathrm{~min}$, they were anesthetized with pentobarbital $(45 \mathrm{mg} / \mathrm{kg})$. Then, the donor's blood vessels and ureter were fully separated. The kidneys were flushed through the aorta with $3 \mathrm{ml}$ of $4^{\circ} \mathrm{C}$ cold Ringer's lactate solution (Shanghai Baxter Healthcare Co., Ltd., Shanghai, China) with heparin (50 units $/ \mathrm{ml}$ ) until homogeneously pale. Only the left kidney of each rat was harvested, leaving the renal arteriovenous, ureter and parts of bladder around the ureter openings intact. Each kidney was placed in cold Ringer's lactate solution at $4^{\circ} \mathrm{C}$ for $180 \mathrm{~min}$. Recipient rats underwent a left nephrectomy prior to accepting the donated kidneys. Orthotopic renal transplantation was then performed. The donor SD rat kidney was implanted orthotopically into a recipient SD rat with end-to-end anastomosis of renal artery, vein and ureter. Finally, recipient rats underwent right nephrectomy. During the surgery, body temperature was monitored and constantly kept between $35^{\circ} \mathrm{C}$ and $37^{\circ} \mathrm{C}$. After transplantation, the rats were placed on a warm blanket with free access to water and standard laboratory chow.
Preservation of kidneys. After all the recipient rats were anesthetized with atropine $(0.01 \mathrm{mg} / \mathrm{kg})$, buprenorphine $(0.04 \mathrm{mg} / \mathrm{kg})$ and diazepam $(10 \mathrm{mg} / \mathrm{kg})$, blood of recipient rats was drawn for analysis $24 \mathrm{~h}$ following kidney transplantation. The renal allografts were harvested for subsequent experiments. While under anesthesia, the animals were sacrificed by cervical dislocation. All rats underwent a laparotomy and nephrectomy to harvest the kidneys. Kidneys were then fixed in $10 \%$ phosphate-buffered formalin or immediately frozen, and stored at $-80^{\circ} \mathrm{C}$ for subsequent experiments.

Serum assays. To assess creatinine $(\mathrm{Cr})$ and blood urea nitrogen (BUN) serum levels, blood samples were collected, centrifuged and kept at $-20^{\circ} \mathrm{C}$ until analysis. The samples were examined using an Olympus AU 2700 Analyzer (Olympus Corporation, Tokyo, Japan), following standard techniques.

Histological examination. The kidney was fixed in $10 \%$ neutral-buffered formalin, embedded in paraffin wax and cut into $4-\mu \mathrm{m}$ thick sections according to standard procedure. Sections were deparaffinzed and hydrated gradually and examined using hematoxylin and eosin staining and immunohistochemistry. Morphological assessment was performed by an experienced renal pathologist who was unaware of the treatment that had been carried out in each case, images were captured using a BX53F light microscope (Olympus Corporation), at a magnification of $\times 400$. A grading scale of $0-4$, as outlined by Jablonski et al (13), was used for the histopathological assessment of isogeneic renal transplantation-induced damage of the proximal tubules.

Periodic acid-Schiff staining. Paraffin sections were routinely dewaxed to water. Serial sections $(4-\mu \mathrm{m})$ were washed with distilled water, incubated in $0.5-1 \%(\mathrm{v} / \mathrm{v})$ aqueous periodate for 5-10 min, and washed a further three times with distilled water. The sections were then incubated in Schiff's reagent (Fuzhou Maixin Biotechnology Development Co., Ltd., Fuzhou, China) for 10-30 min. After staining, the sections were washed three times with sulfite, then Rinsed with running water for $10 \mathrm{~min}$, and then washed with distilled water once. The sections were then counterstained with hematoxylin to identify nuclei.

Terminal deoxynucleotidyl transferase dUTP nick end labeling (TUNEL) assay. To observe cell apoptosis induced by ischemia, an in situ apoptosis detection kit (Promega Corporation, Madison, WI, USA) was used. The TUNEL assay was performed based on the manufacturer's instructions. Briefly, after being fixed in $4 \%$ paraformaldehyde/phosphate-buffered saline (PBS; pH 7.4) solution at $4^{\circ} \mathrm{C}$ overnight, the whole specimens were extensively washed with $1 \mathrm{X}$ PBS solution three times, then immersed into $70 \%$ ethanol for at least $24 \mathrm{~h}$ at $20^{\circ} \mathrm{C}$. After being washed a further three times with PBS solution, the samples were immersed into a permeabilization buffer for $15 \mathrm{~min}$ on ice then washed again with PBS solution. Subsequently they were incubated in $50 \mathrm{ml}$ reaction buffer ( $5 \mathrm{ml}$ terminal deoxynucleotidyl transferase enzyme and $45 \mathrm{ml}$ Labeling Safe Buffer) (Promega Corporation) for $90 \mathrm{~min}$ at $37^{\circ} \mathrm{C}$. The labeling procedure was stopped by washing with PBS solution. The image was analyzed using a Zeiss LSM 
510 Confocal laser scanning microscope (Carl Zeiss AG, Oberkoden, Germany) with a $488 \mathrm{~nm}$ excitation line and a $530 \mathrm{~nm}$ emission filter. Five high-power fields of vision in the distribution areas of the apoptotic cells in each slide were randomly selected, and the average number of apoptotic cells per 100 cells was calculated. The apoptotic index (AI) was expressed as a percentage.

Measurement of malondialdehyde (MDA), superoxide dismutase (SOD), glutathione (GSH) and catalase (CAT) levels in the kidney. Renal tissue MDA concentration was measured using the thiobarbituric acid method. Levels of lipid peroxides were measured as an indicator of MDA production rates (assay kit; Nanjing Jiancheng Bioengineering Institute, Nanjing, China). Absorbance was measured at $532 \mathrm{~nm}$ using a spectrometer. SOD activity in renal tissue was measured using a commercialized chemical assay kit (Nanjing Jiancheng Bioengineering Institute) by the xanthine oxidase method. Absorbance was determined at $550 \mathrm{~nm}$ using a spectrometer. GSH content was detected using a colorimetric GSH detection kit (Nanjing Jiancheng Bioengineering Institute). Briefly, the renal homogenate was mixed with reagent $\mathrm{A}$, reagent $\mathrm{B}$ and reagent $\mathrm{C}$. Absorbance of the yellow solution was measured at $412 \mathrm{~nm}$. CAT activity in renal tissue was measured using a commercialized chemical assay kit (Nanjing Jiancheng Bioengineering Institute) by the visible light method. Absorbance was determined at $405 \mathrm{~nm}$ using a spectrometer. All protein concentrations of renal tissue homogenate samples were determined using the Coomassie Blue method (assay kit; Nanjing Jiancheng Bioengineering Institute).

Immunohistochemistry. The expression levels of nuclear factor erythroid 2-related factor 2 (Nrf-2) and heme oxygenase 1 (HO-1) were evaluated using immunohistochemical staining. Briefly, 5- $\mu \mathrm{m}$ sections were deparaffinized, and endogenous peroxidase activity was blocked with $3 \%$ hydrogen peroxide at $37^{\circ} \mathrm{C}$ for $10 \mathrm{~min}$. Then, the sections were treated with $10 \%$ normal goat serum (Wuhan Boster Biological Technology, Ltd., Wuhan, China) in Tris-buffered saline for $30 \mathrm{~min}$ at $37^{\circ} \mathrm{C}$. Subsequently, they were incubated overnight at $4^{\circ} \mathrm{C}$ with a rabbit polyclonal anti-rat antibody to $\mathrm{Nrf}-2$ (catalogue no. sc-722, 1:300; Santa Cruz Biotechnology, Inc., Dallas, TX, USA) and a rabbit polyclonal anti-rat antibody to HO-1 (catalogue no. 10701-1-AP, 1:1,000; Wuhan Sanying Biotechnology; Proteintech Group Inc., Wuhan, China). After washing three times with PBS, these sections were incubated with the horseradish peroxidase (HRP)-conjugated anti-rabbit secondary antibody (catalogue no. BA1054, 1:5,000, Wuhan Boster Biological Technology, Ltd., Wuhan, China) for $30 \mathrm{~min}$ at room temperature, after which the color reagent 3,3'-diaminobenzidine was added. For the negative control group, the same procedures were performed with the exception of adding the primary antibody.

Western blot analysis. Proteins were extracted and purified from renal tissue as previously described (14). In brief, protein samples were prepared for gel electrophoresis and separated on $12.5 \%$ sodium dodecyl sulfate-polyacrylamide gels (40 $\mu \mathrm{g} /$ lane), then transferred to a nitrocellulose membrane
(Bio-Rad Laboratories, Inc., Hercules, CA, USA). The membrane was blocked with 5\% nonfat dry milk in Tris-buffered saline with Tween (TBST) buffer, then incubated with primary antibodies overnight at $4^{\circ} \mathrm{C}$. After rinsing with TBST buffer extensively, the blots were incubated with secondary antibodies, and developed with the use of an enhanced chemiluminescence kit (Pierce Protein Biology; Thermo Fisher Scientific, Inc., Waltham, MA, USA). Finally, the blots were captured on light-sensitive imaging film (Kodak, Rochester, NY, USA) for analysis. The following antibodies were used: A rabbit polyclonal antibody to Nrf-2 (catalogue no. sc-722, 1:300; Santa Cruz Biotechnology, Inc., Dallas, TX, USA), a rabbit polyclonal antibody to HO-1 (catalogue no. 10701-1-AP, 1:1,000; Wuhan Sanying Biotechnology, Wuhan, China), and a rabbit polyclonal antibody to GAPDH (catalogue no. BA-2913, 1:300; Wuhan Boster Biological Technology, Ltd., Wuhan, China). GAPDH was used to show equal amounts of protein loading in each lane. HRP-conjugated anti-rabbit (BA-1054, 1:5,000; Wuhan Boster Biological Technology, Ltd., Wuhan, China) or anti-mouse secondary antibodies (BA-1051, 1:5,000, Wuhan Boster Biological Technology, Ltd., Wuhan, China). The expression levels following the western blot analysis of each protein was assessed for the three groups using ImageJ software. To quantify the results, the average gray value of each protein was calculated.

Statistical analysis. All the data were statistically analyzed using SPSS 18.0 statistical software (SPSS Inc., Chicago, IL, USA) and presented as the mean \pm standard deviation. Statistical differences were analyzed using the Student's $t$-test and $\mathrm{P}<0.05$ was considered to indicate a statistically significant difference.

\section{Results}

Effect of OOP on renal function after kidney transplantation. The renal functional parameters of rats were detected $24 \mathrm{~h}$ after renal transplantation. Rats subjected to isogeneic renal transplantation showed a significant increase in BUN and $\mathrm{Cr}$ levels compared with sham-operated rats $(\mathrm{P}<0.05)$. The negative effects on renal function induced by renal transplantation were significantly reduced in the $\mathrm{OOP}+\mathrm{KT}$ group compared with the KT group, in relation to levels of BUN or serum $\mathrm{Cr}$ $(\mathrm{P}<0.05$; Fig. 1).

Effect of OOP on morphological features of renal cells. Histopathological examination revealed that morphological lesions existed in the allograft kidney tissue after isogeneic renal transplantation. Renal injury was evidenced by loss of brush borders, congestion, tubular cell swelling and tubular dilation. However, this renal damage was attenuated by OOP (Figs. 2 and 3). Furthermore, the Jablonski grade analysis of severe acute tubular necrosis results showed that the OOP+KT group had significantly lower levels of damage compared with the KT group (Table I). In addition, OOP significantly reduced the $\mathrm{AI}$ in the $\mathrm{OOP}+\mathrm{KT}$ group compared with the KT group $(\mathrm{P}<0.05$; Table I and Fig. 4).

Levels of SOD, MDA, GSH and CAT in renal tissue. As shown in Table II, the level of MDA content, which was indicated 
Table I. Effect of OOP on Jablonski grade and apoptosis index in renal tissue $(n=6)$.

\begin{tabular}{lcrr}
\hline Group & $\mathrm{S}$ & $\mathrm{KT}$ & $\mathrm{OOP}+\mathrm{KT}$ \\
\hline Jablonski grade & $0.31 \pm 0.27$ & $3.16 \pm 0.35^{\mathrm{a}}$ & $2.43 \pm 0.29^{\mathrm{a}, \mathrm{b}}$ \\
Apoptosis index, \% & $1.58 \pm 0.35$ & $27.45 \pm 2.46^{\mathrm{a}}$ & $17.80 \pm 2.73^{\mathrm{a}, \mathrm{b}}$ \\
\hline
\end{tabular}

Data are presented as the mean \pm standard deviation. ${ }^{\mathrm{a}} \mathrm{P}<0.05$ vs. $\mathrm{S}$ group, ${ }^{\mathrm{b}} \mathrm{P}<0.05$ vs. KT group. $\mathrm{S}$, sham-operated; KT, kidney transplantation; $\mathrm{OOP}+\mathrm{KT}$, ozone oxidative preconditioning and kidney transplantation.

Table II. Effect of OOP on the protein expression levels of SOD, MDA, GSH and CAT in renal tissue $24 \mathrm{~h}$ after renal transplantation $(n=6)$.

\begin{tabular}{llccc}
\hline Groups & SOD, $\mathrm{U} / \mathrm{mg}$ & MDA, nmol/mg & GSH, nmol/mg & CAT, U/mg \\
\hline S & $85.83 \pm 7.24$ & $3.40 \pm 1.30$ & $10.10 \pm 0.94$ & $71.93 \pm 6.43$ \\
KT & $39.36 \pm 8.23^{\mathrm{a}}$ & $6.26 \pm 1.87^{\mathrm{a}}$ & $3.29 \pm 1.65^{\mathrm{a}}$ & $35.75 \pm 5.93^{\mathrm{a}}$ \\
OOP+KT & $57.42 \pm 9.02^{\mathrm{a}, \mathrm{b}}$ & $4.83 \pm 1.92^{\mathrm{a}, \mathrm{b}}$ & $7.46 \pm 1.49^{\mathrm{a}, \mathrm{b}}$ & $57.55 \pm 7.31^{\mathrm{a}, \mathrm{b}}$ \\
\hline
\end{tabular}

Data are presented as the mean \pm standard error. ${ }^{a} \mathrm{P}<0.05$ vs. $\mathrm{S}$ group, ${ }^{\mathrm{b}} \mathrm{P}<0.05$ vs. KT group. $\mathrm{S}$, sham-operated; KT, kidney transplantation; $\mathrm{OOP}+\mathrm{KT}$, ozone oxidative preconditioning and kidney transplantation; SOD, superoxide dismutase; MDA, malondialdehyde; GSH, glutathione; CAT, catalase.
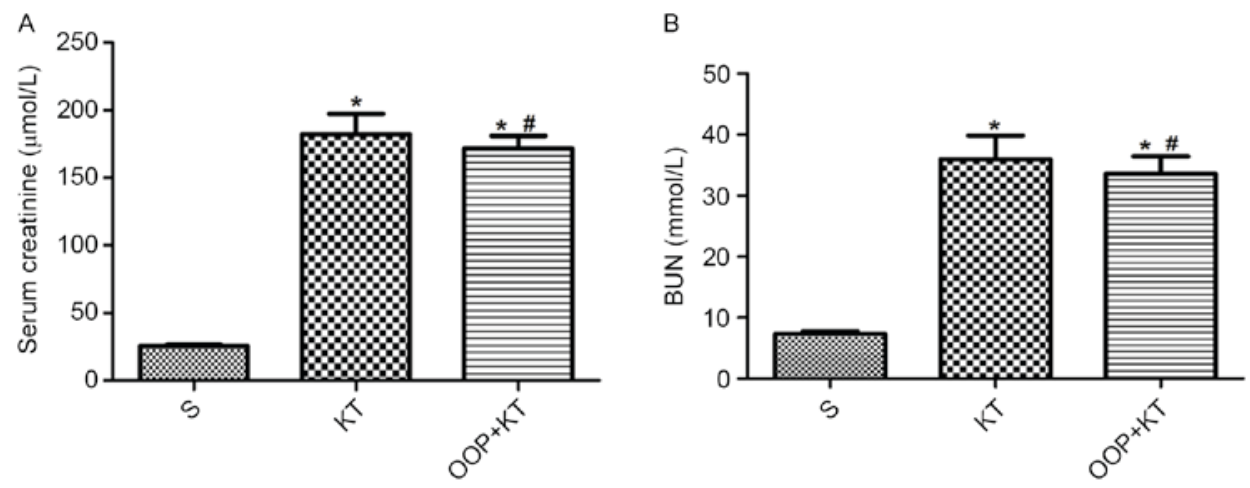

Figure 1. Effect of OOP on renal function after kidney transplantation. Renal function is measured in terms of (A) serum Cr concentration and (B) serum BUN concentration following kidney transplantation and $24 \mathrm{~h}$ of reperfusion. Bars represent the mean \pm standard deviation ( $\mathrm{n}=6$ ). ${ }^{*} \mathrm{P}<0.05$, vs. $\mathrm{S}$ group; ${ }^{\sharp} \mathrm{P}<0.05$, vs. KT group. OOP, ozone oxidative preconditioning; S, sham; KT, kidney transplantation; OOP+KT, ozone oxidative preconditioning and kidney transplantation; $\mathrm{Cr}$, creatinine; BUN, blood urea nitrogen.

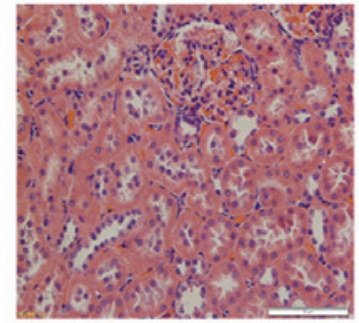

S group

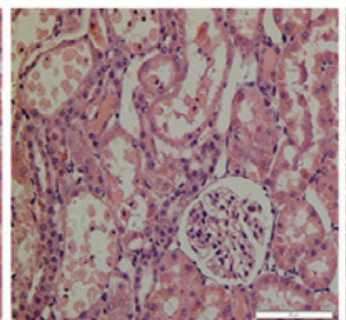

KT group

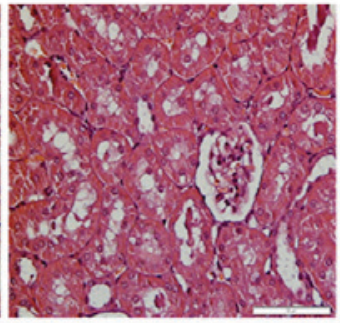

OOP+KT group

Figure 2. Effect of OOP on morphological lesions in renal cells. Morphological lesions after renal transplantation were studied using hematoxylin and eosin staining. Original magnification $\mathrm{x} 400$. Scale bar, $50 \mu \mathrm{m}$. S, sham; KT, kidney transplantation; OOP+KT, ozone oxidative preconditioning and kidney transplantation.

by the level of lipid peroxidation, was significantly higher in the KT and OOP+KT groups than in the $\mathrm{S}$ group $(\mathrm{P}<0.05)$.
However, the level of MDA in the OOP+KT group was significantly lower than in the KT group $(\mathrm{P}<0.05)$. Meanwhile, the 


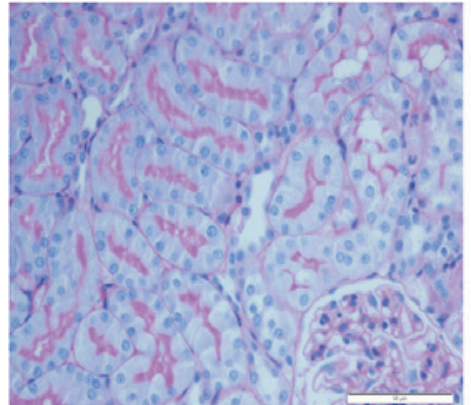

$\mathrm{S}$ group

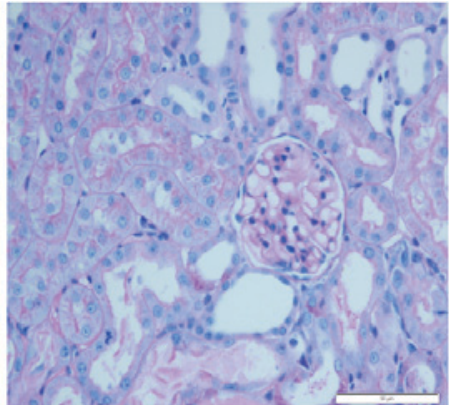

KT group

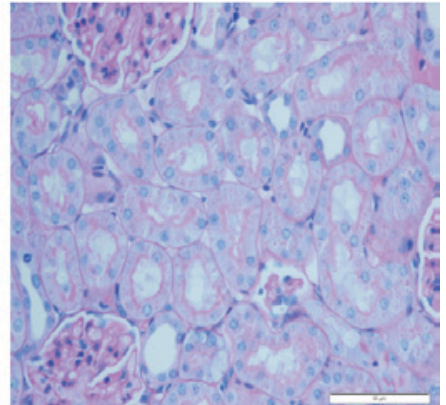

OOP + KT group

Figure 3. Effect of OOP on renal tubular brush borders. Destruction of renal tubular brush borders after renal transplantation was detected using periodic acid-Schiff staining. Original magnification, $\mathrm{x} 400$. Scale bar, $50 \mu \mathrm{m}$. S, sham; KT, kidney transplantation; OOP+KT, ozone oxidative preconditioning and kidney transplantation.

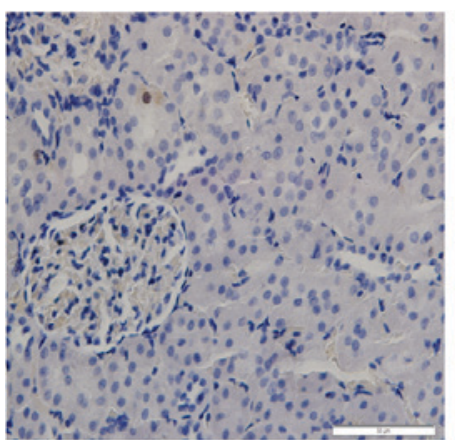

$\mathrm{S}$ group

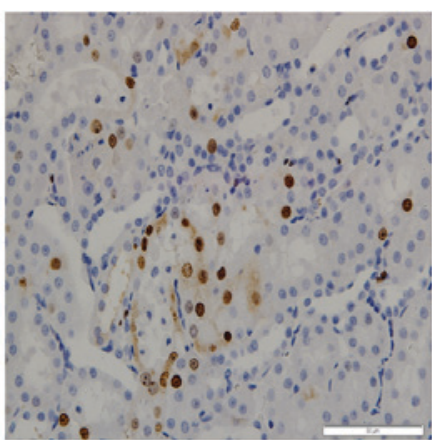

KT group

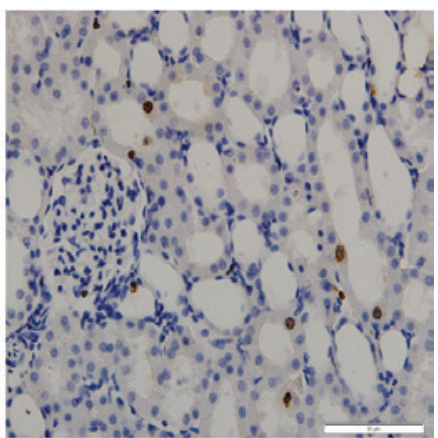

OOP + KT group

Figure 4. Effect of OOP on renal tubular epithelial cell apoptosis after kidney transplantation. The occurrence of apoptosis in renal tubular epithelial cells was studied using a TUNEL assay. Original magnification, $\mathrm{x} 400$. Scale bar, $50 \mu \mathrm{m}$. S, sham; KT, kidney transplantation; OOP+KT, ozone oxidative preconditioning and kidney transplantation; TUNEL, terminal deoxynucleotidyl transferase dUTP nick end labeling.

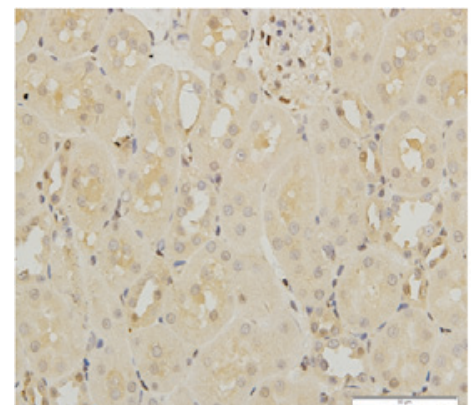

$\mathrm{S}$ group

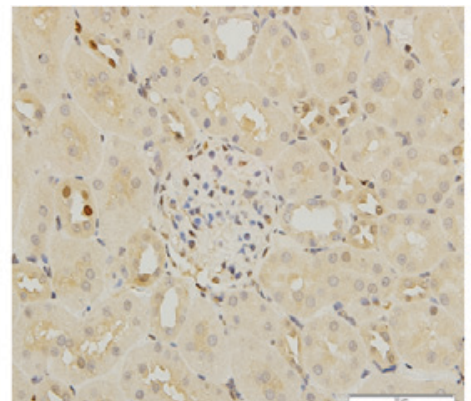

KT group

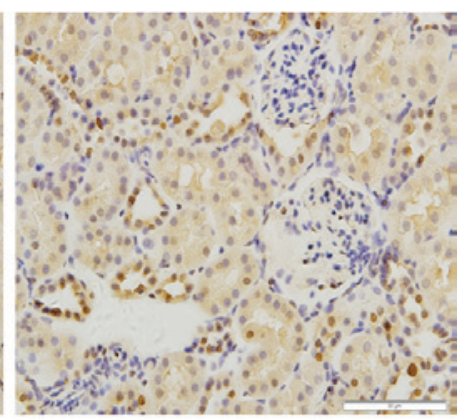

$\mathrm{OOP}+\mathrm{KT}$ group

Figure 5. Expression levels of Nrf-2 in renal tissue revealed by immunohistochemistry. Original magnification, x400. Scale bar, 50 $\mu$ m. Nrf-2, nuclear factor erythroid 2-related factor 2; S, sham; KT, kidney transplantation; OOP+KT, ozone oxidative preconditioning and kidney transplantation.

levels of SOD, GSH and CAT in the kidney tissue were significantly decreased after kidney transplantation in the KT group and the OOP+KT group compared with the $\mathrm{S}$ group $(\mathrm{P}<0.05)$. However, the levels of SOD, GSH and CAT after kidney transplantation were significantly higher in the OOP+KT group compared with the KT group $(\mathrm{P}<0.05)$.

Expression levels of $\mathrm{Nrf}-2$ and $\mathrm{HO}-1$. The results of the immunohistochemistry analysis showed that the expression levels of Nrf-2 and HO-1 in the OOP+KT group were higher than those in the KT group (Figs. 5 and 6). Furthermore, the western blot analysis confirmed that the expression levels of Nrf-2 and HO-1 in the OOP+KT group were significantly higher than those in the KT group $(\mathrm{P}<0.05$; Fig. 7).

\section{Discussion}

Compared with dialysis treatment, kidney transplantation is often seen as preferable for patients with end-stage kidney disease, and previous research confirms that renal transplant patients have a longer survival time, with a better quality of life, than dialysis patients $(15,16)$. However, despite a low 


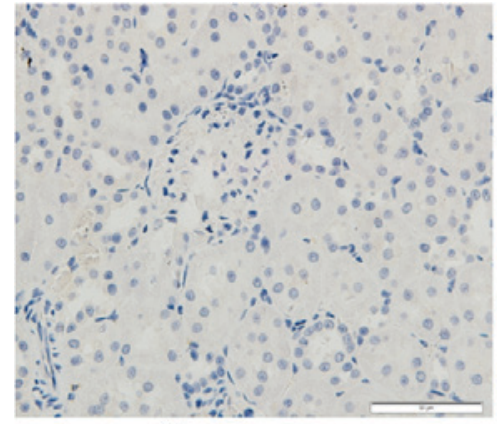

$\mathrm{S}$ group

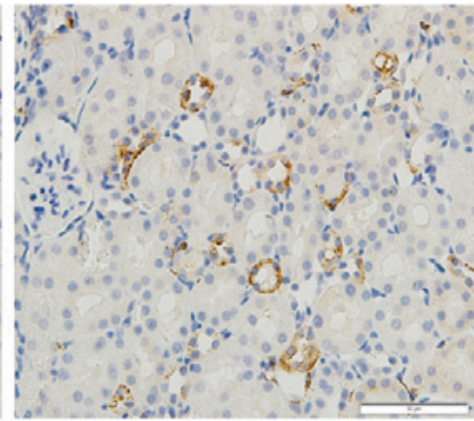

KT group

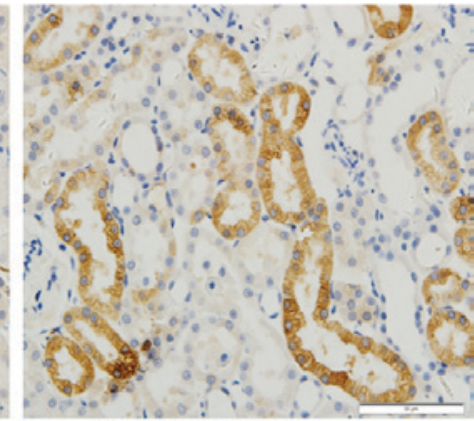

$\mathrm{OOP}+\mathrm{KT}$ group

Figure 6. Expression levels of HO-1 in renal tissue revealed by immunohistochemistry. Original magnification, $\mathrm{x} 400$. Scale bar, $50 \mu \mathrm{m}$. HO-1, heme oxygenase 1; $\mathrm{S}$, sham; KT, kidney transplantation; OOP+KT, ozone oxidative preconditioning+ kidney transplantation.
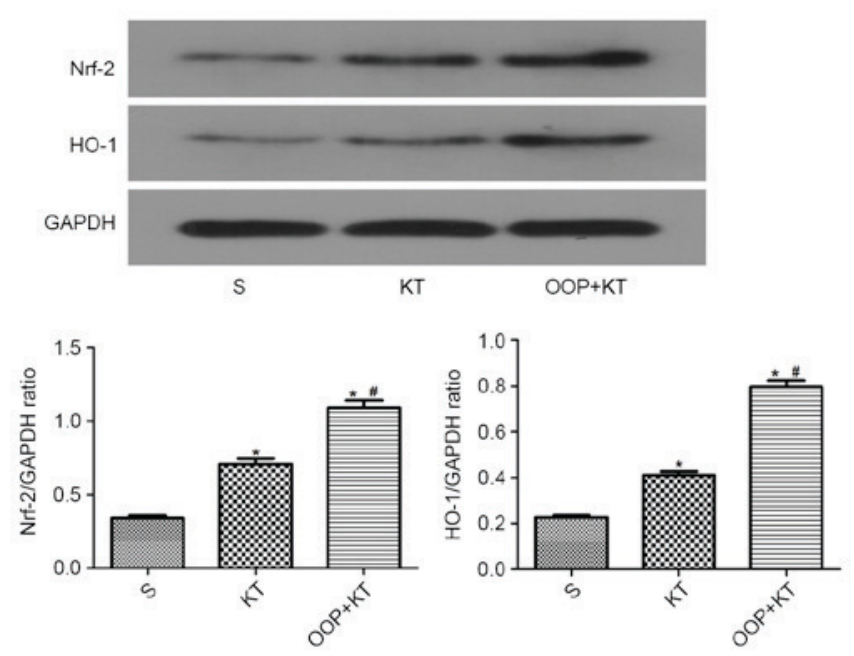

Figure 7. Expression levels of Nrf-2 and HO-1 in renal tissue following renal transplantation and $24 \mathrm{~h}$ of reperfusion, revealed by western blot analysis. Bars represent the mean \pm standard deviation $(n=6) .{ }^{*} \mathrm{P}<0.05$, vs. $\mathrm{S}$ group; ${ }^{\#} \mathrm{P}<0.05$, vs. KT group. Nrf-2, nuclear factor erythroid 2-related factor 2; HO-1, heme oxygenase 1; GAPDH, glyceraldehyde 3-phosphate dehydrogenase; S, sham; KT, kidney transplantation; OOP+KT, ozone oxidative preconditioning and kidney transplantation.

occurrence of acute allograft rejection due to the application of novel anti-immunity drugs, some detrimental factors still exist during the perioperative period of renal transplantation, such as IRI and chronic graft rejection (17).

IRI is a pathological process that involves oxidative stress, intracellular calcium overloading, inflammation reaction and cell apoptosis. Oxidative stress is one of the most important components of IRI, and is primarily caused by excessive reactive oxygen species (ROS) being generated in ischemic tissue after reperfusion (18). Naturally generated antioxidant enzymes can counteract the cellular effects of oxygen free radicals under normal conditions $(18,19)$, but excessive ROS generation during the period of ischemia reperfusion cannot be effectively controlled by this system (20). It has been reported that inflammation cytokines and chemokines are released during oxygen radical generation and lipid peroxidation. Together with adhesion molecules, these attract inflammatory cells, such as monocytes and neutrophils, which in turn help to release more ROS and aggravate renal damage (2). Therefore, effective measures or treatments to attenuate oxidative stress or help balance the oxidative and anti-oxidative systems may be useful in alleviating IRI during renal transplantation.

In the present study, the effects of OOP on kidney transplantation were investigated in a rat model. It was found that pretreatment with a controlled concentration of ozone before kidney transplantation could decrease oxidative stress injury, demonstrated by a significantly reduced renal tissue concentration of MDA in the OOP+KT group compared with the KT group. Furthermore, compared with the KT group, indicators of anti-oxidative stress significantly increased in the allograft kidney tissue, such as SOD, GSH and CAT. These results were consistent with a prior study, which identified that OOP improves organ stress by enhancing endogenous protective mechanisms (21).

Nrf2 belongs to the cap ' $n$ ' collar family, a small group of transcription factors that contain a unique basic leucine zipper motif $(22,23)$. Nrf2 principally regulates transcriptional activation through an antioxidant responsive element (ARE) (24), which is considered to be a cis-acting regulatory element in promoter regions of many cytoprotective genes. Under basal conditions, Nrf2 is constantly targeted for Keap-1-mediated ubiquitination and subsequent proteasomal degradation to maintain low Nrf2 protein levels (25). However, upon activation, Nrf2 can activate downstream antioxidant proteins, phase II metabolizing/detoxifying enzymes and phase III ATP-dependent drug efflux pump-encoded genes (26-28). It has been reported that Nrf2 activates numerous types of enzymes with antioxidation and detoxifying activities that serve a key function in the protection of cells against various environmental stresses, such as electrophiles, ROS and reactive nitrogen species (29). In the present study, the expression level of Nrf2 in the OOP+KT group was significantly higher than that in the KT group. A proposed explanation for this is the low levels of OOP in the KT group, which could have helped to trigger the activation of Nrf2/Keap-1. Furthermore, a prior study indicates that overexpression of Nrf2 increases ARE transcriptional activity and enhances the expression of several ARE-dependent antioxidant and cytoprotective enzymes, including HO-1, glutamate cysteine ligase, glutathione peroxidase and $\mathrm{NAD}(\mathrm{P}) \mathrm{H}$ quinone oxidoreductase 1 (30). In the present study, the expression level of $\mathrm{HO}-1$ in the OOP+KT group was significantly higher than that in the KT group, which could help to explain why the OOP+KT group displayed less severe oxidative stress injury than the KT group. In 
addition, it has previously been proposed that Nrf2-dependent HO-1 expression could inhibit the activation of nuclear factor $\kappa \mathrm{B}$, stimulated by tumor necrosis factor alpha and monocyte chemoattractant protein-1 secretion in endothelial cells (31).

In addition, the present results suggest that OOP could reduce the rate of apoptosis in renal tubular epithelium cells caused by oxidative stress injury during the process of renal transplantation. It has been demonstrated that excessive ROS overwhelming the scavenging capacity of the endogenous antioxidant system can lead to cellular damage (32), such as blocking cellular mitochondrial respiration (33), or facilitating the formation of mitochondrial transition pores, which help to increase the release of apoptosis-related proteins after ischemia reperfusion $(34,35)$. A previous study outlines several measures to prevent oxidative stress-induced apoptosis, such as increasing the activity of the endogenous antioxidant system, applying exogenous antioxidant enzymes or free radical scavengers, or decreasing the lipid peroxidation byproducts (36). In this study, it was indicated that OOP reduces the apoptosis or necrosis of renal tubular epithelium cells, which may be related to the strengthening of endogenous antioxidant systems, such as the $\mathrm{Nrf} 2 / \mathrm{HO}-1$ pathway.

In conclusion, this study demonstrated that OOP could mitigate oxidative stress injury and apoptosis of renal tubular epithelium cells during kidney transplantation in a rat model, which may be related to activation of the Nrf2/HO-1 signaling pathway and reduction of renal tubular epithelial cell apoptosis. However, the long-term effect of OOP on renal transplantation has not been studied here, such as the differences in survival rate between groups. Furthermore, the effects of OOP on the immune response and inflammation between different strains of rats, especially in the acute immunological rejection model, need to be investigated further.

\section{Acknowledgements}

This study was supported by the National Natural Science Foundation of China (grant no. 81400753) and the Natural Science Foundation of Hubei Province (grant no. 2014CFB362).

\section{References}

1. Wolfe RA, Ashby VB, Milford EL, Ojo AO, Ettenger RE, Agodoa LY, Held PJ and Port FK: Comparison of mortality in all patients on dialysis, patients on dialysis awaiting transplantation, and recipients of a first cadaveric transplant. N Engl J Med 341: $1725-1730,1999$

2. Perico N, Cattaneo D, Sayegh MH and Remuzzi G: Delayed graft function in kidney transplantation. Lancet 364: 1814-1827, 2004.

3. Peeters P, Terryn W, Vanholder R and Lameire N: Delayed graft functioning in renal transplantation. Curr Opin Crit Care 10: 489-498, 2004.

4. Gulmen S, Kurtoglu T, Meteoglu I and Okutan H: Ozone therapy as an adjunct to vancomycin enhances bacterial elimination in methicillin resistant Staphylococcus aureus mediastinitis. J Surg Res 185: 64-69, 2013.

5. Bocci V: Is it true that ozone is always toxic? The end of a dogma. Toxicol Appl Pharmacol 216: 493-504, 2006.

6. Sagai M and Bocci V: Mechanisms of action involved in ozone therapy: Is healing induced via a mild oxidative stress? Med Gas Res 1: 29, 2011.

7. Bocci VA: Scientific and medical aspects of ozone therapy. State of the art. Arc Med Res 37: 425-435, 2006.

8. Inal M, Dokumacioglu A, Ozcelik E and Ucar O: The effects of ozone therapy and coenzyme Q10 combination on oxidative stress markers in healthy subjects. Ir J Med Sci 180: 703-707, 2011.
9. Guven A, Gundogdu G, Sadir S, Topal T, Erdogan E, Korkmaz A, Surer I and Ozturk H: The efficacy of ozone therapy in experimental caustic esophageal burn. J Pediatr Surg 43: 1679-1684, 2008.

10. Bakkal BH, Gultekin FA, Guven B, Turkcu UO, Bektas S and Can M: Effect of ozone oxidative preconditioning in preventing early radiation-induced lung injury in rats. Braz J Med Biol Res 46: 789-796, 2013.

11. Chen H, Xing B, Liu X, Zhan B, Zhou J, Zhu H and Chen Z: Ozone oxidative preconditioning protects the rat kidney from reperfusion injury: The role of nitric oxide. J Surg Res 149: 287-295, 2008.

12. Ajamieh HH, Menéndez S, Martínez-Sánchez G, Candelario-Jalil E, Re L, Giuliani A and Fernández OS: Effects of ozone oxidative preconditioning on nitric oxide generation and cellular redox balance in a rat model of hepatic ischaemia-reperfusion. Liver Int 24: 55-62, 2004

13. Jablonski P, Howden BO, Rae DA, Birrell CS, Marshall VC and Tange J: An experimental model for assessment of renal recovery from warm ischemia. Transplantation 35: 198-204, 1983.

14. Park KM, Cho HJ and Bonventre JV: Orchiectomy reduces susceptibility to renal ischemic injury: A role for heat shock proteins. Biochem Biophys Res Commun 328: 312-317, 2005.

15. Laupacis A, Keown P, Pus N, Krueger H, Ferguson B, Wong C and Muirhead N: A study of the quality of life and cost-utility of renal transplantation. Kidney Int 50: 235-242, 1996.

16. Schnuelle P, Lorenz D, Trede M and Van Der Woude FJ: Impact of renal cadaveric transplantation on survival in end-stage renal failure: Evidence for reduced mortality risk compared with hemodialysis during long-term follow-up. J Am Soc Nephrol 9; 2135-2141, 1998.

17. Menke J, Sollinger D, Schamberger B, Heemann U and Lutz J: The effect of ischemia/reperfusion on the kidney graft. Curr Opin Organ Transplant 19: 395-400, 2014.

18. Haugen E and Nath KA: The involvement of oxidative stress in the progression of renal injury. Blood Purif 17: 58-65, 1999.

19. Edelstein CL, Ling H and Schrier RW: The nature of renal cell injury. Kidney Int 51: 1341-1351, 1997.

20. Castaneda MP, Swiatecka-Urban A, Mitsnefes MM, Feuerstein D, Kaskel FJ, Tellis V and Devarajan P: Activation of mitochondrial apoptotic pathways in human renal allografts after ischemia reperfusion injury. Transplantation 76: 50-54, 2003

21. Rodriguez ZZ, Guanche D, Alvarez RG, Rosales FH, Alonso Y and Schulz S: Preconditioning with ozone/oxygen mixture induces reversion of some indicators of oxidative stress and prevents organic damage in rats with fecal peritonitis. Inflamm Res 58: 371-375, 2009.

22. Itoh $\mathrm{K}$, Igarashi $\mathrm{K}$, Hayashi $\mathrm{N}$, Nishizawa $\mathrm{M}$ and Yamamoto $\mathrm{M}$ : Cloning and characterization of a novel erythroid cell-derived CNC family transcription factor heterodimerizing with the small Maf family proteins. Mol Cell Biol 15: 4184-4193, 1995.

23. Sykiotis GP and Bohmann D: Stress-activated cap'n'collar transcription factors in aging and human disease. Sci Signal 3: re3, 2010.

24. Huang HC, Nguyen T and Pickett CB: Phosphorylation of Nrf2 at Ser-40 by protein kinase $\mathrm{C}$ regulates antioxidant response element-mediated transcription. J Biol Chem 277: 42769-42774, 2002.

25. Chang KM, Chen HH, Wang TC, Chen IL, Chen YT, Yang SC, Chen YL, Chang HH, Huang CH, Chang JY, et al: Novel oxime-bearing coumarin derivatives act as potent Nrf2/ARE activators in vitro and in mouse model. Eur J Med Chem 106: 60-74, 2015.

26. Giudice A and Montella M: Activation of the Nrf2-ARE signaling pathway: A promising strategy in cancer prevention. Bioessays 28: 169-181, 2006.

27. Maher JM, Dieter MZ, Aleksunes LM, Slitt AL, Guo G, Tanaka Y, Scheffer GL, Chan JY, Manautou JE, Chen Y, et al: Oxidative and electrophilic stress induces multidrug resistance-associated protein transporters via the nuclear factor-E2-related factor-2 transcriptional pathway. Hepatology 46: 1597-1610, 2007.

28. Zhang Y and Gordon GB: A strategy for cancer prevention: Stimulation of the Nrf2-ARE signaling pathway. Mol Cancer Ther 3: 885-893, 2004.

29. Cominacini L, Mozzini C, Garbin U, Pasini A, Stranieri C, Solani E, Vallerio P, Tinelli IA and Fratta Pasini A: Endoplasmic reticulum stress and Nrf2 signaling in cardiovascular diseases. Free Radic Biol Med 88: 233-242, 2015.

30. Aboonabi A and Singh I: Chemopreventive role of anthocyanins in atherosclerosis via activation of Nrf2-ARE as an indicator and modulator of redox. Biomed Pharmacother 72: 30-36, 2015. 
31. Osburn WO, Karim B, Dolan PM, Liu G, Yamamoto M, Huso DL and Kensler TW: Increased colonic inflammatory injury and formation of aberrant crypt foci in Nrf2-deficient mice upon dextran sulfate treatment. Int J Cancer 121: 1883-1891, 2007.

32. Chong ZZ, Li F and Maiese K: Oxidative stress in the brain: Novel cellular targets that govern survival during neurodegenerative disease. Prog Neurobiol 75: 207-246, 2005.

33. Yamamoto T, Maruyama W, Kato Y, Yi H, Shamoto-Nagai M, Tanaka M, Sato Y and Naoi M: Selective nitration of mitochondrial complex I by peroxynitrite: Involvement in mitochondria dysfunction and cell death of dopaminergic SH-SY5Y cells. J Neural Transm (Vienna) 109: 1-13, 2002.

34. Kim GW, Kondo T, Noshita N and Chan PH: Manganese superoxide dismutase deficiency exacerbates cerebral infarction after focal cerebral ischemia/reperfusion in mice: Implications for the production and role of superoxide radicals. Stroke 33: 809-815, 2002.
35. Murakami K, Kondo T, Kawase M, Li Y, Sato S, Chen SF and Chan PH: Mitochondrial susceptibility to oxidative stress exacerbates cerebral infarction that follows permanent focal cerebral ischemia in mutant mice with manganese superoxide dismutase deficiency. J Neurosci 18: 205-213, 1998.

36. Huang T, Cheng AG, Stupak H, Liu W, Kim A, Staecker H, Lefebvre PP, Malgrange B, Kopke R, Moonen G and Van De Water TR: Oxidative stress-induced apoptosis of cochlear sensory cells: Otoprotective strategies. Int J Dev Neurosci 18: 259-270, 2000. 